\title{
Phase Similarity Index for Image Quality Assessment
}

\author{
Huawen Chang ${ }^{\mathrm{a}, *}$, Changwei Mao ${ }^{\mathrm{a}}$, and Minghui Wang ${ }^{\mathrm{b}}$ \\ ${ }^{a}$ School of Computer and Communication Engineering, Zhengzhou University of Light Industry, Zhengzhou, 450001, China \\ ${ }^{b}$ College of Computer Science, Sichuan University, Chengdu, 610064, China
}

\begin{abstract}
Image quality evaluation is significant in many fields of image applications and research, such as the performance evaluation of image processing algorithms, image compression, encoding, and transmission. This study aims to design an evaluation method by using the phase and structure information of images. Compared with the amplitude structure of the image, the visual system is more sensitive to phase information. Based on this premise, a new image quality assessment method, which is named the phase similarity index (PSI), is proposed. It considers three important features for visual perception: brightness, contour, and phase information. To test the performance of PSI, relevant experiments are conducted on TID2008, TID2013, and CSIQ databases. The experimental results show that PSI can achieve higher consistency with subjective evaluation compared with the most advanced image quality metrics.
\end{abstract}

Keywords: image quality assessment; phase characteristic; human visual system (HVS)

(Submitted on September 11, 2019; Revised on October 15, 2019; Accepted on November 27, 2019)

(C) 2019 Totem Publisher, Inc. All rights reserved.

\section{Introduction}

In the process of acquisition, processing, compression, and transmission, images are often interfered by various irrelevant signals, resulting in distortion of the final image. In order to ensure the output quality of these image systems or processing algorithms, it is necessary to have a set of reliable quality evaluation criteria to distinguish the quality of images. Therefore, image quality assessment (IQA) plays an important role in many image processing fields, such as evaluating the advantages and disadvantages of image processing algorithms, assisting detection and proofreading image systems, and optimizing image processing algorithms.

Since human visual systems are the final judges of image quality, the only reliable evaluation method at present is the subjective quality evaluation method, which determines the quality of an image through the evaluation of a large number of human observers. The advantage of the subjective quality evaluation method is that it can produce accurate, reliable, and completely consistent evaluation results with human subjective feelings. However, due to the need for human participation, the implementation of the subjective evaluation method is too costly, time-consuming, and laborious in operation, and it cannot be applied in real-time systems at all. Therefore, many studies focus on objective quality evaluation in order to design an image quality evaluation algorithm that can automatically and efficiently calculate results close to human evaluation. The purpose of objective image quality evaluation is to design a quality evaluation algorithm that is consistent with human subjective perception results.

In the objective image quality evaluation research field, the objective image quality evaluation method is generally divided into three kinds according to the reference image information: full reference, no reference, and reduced reference [1-2]. Full reference quality evaluation is involved in the assessment of the quality of a given image distortion, and perception without distortion of the image is used as a reference to assist in the evaluation. Such a "perfect" image is often referred to as a reference image. No reference quality evaluation is the evaluation of image quality with no information about the image for comparison and reference. Reduced reference quality evaluation involves reducing information about the reference image. For example, in the evaluation of fusion results of multi-spectral and panchromatic remote sensing images, although

\footnotetext{
* Corresponding author.

E-mail address: changhuawen@gmail.com
} 
there is no final perfect fusion result image for reference, part of the best quality information of fusion results can be obtained indirectly from the source images involved in fusion.

Among various image quality evaluation methods, the methods based on the peak signal-to-noise ratio (PSNR) and mean squared error (MSE) have been widely used due to their simplicity and high efficiency. However, they cannot reflect the subjective perception characteristics of the image of the human visual system and ignore the edge, direction, and geometric features of the image itself. They also do not have good subjective and objective consistency. Therefore, combined with the principle of human visual systems, many researchers have proposed effective objective evaluation algorithms for image quality.

Some researchers have discussed the subjective and objective evaluation methods of images and emphatically introduced the objective evaluation methods of image quality from a single viewpoint. They analyzed the advantages and disadvantages of existing image quality evaluation methods and proposed several research directions for the further study of image quality evaluation methods [3]. Sampat proposed a wavelet domain image quality evaluation method based on structural similarity, and the experimental results showed that this algorithm is more consistent with the visual perception characteristics of human eyes than the structural similarity algorithm [4]. Chandler proposed an image quality evaluation algorithm based on wavelets and structure information. Based on the visual perception of image and the wavelet transform, an image quality model of visual signal-to-noise ratio (VSNR) was proposed [5]. Gao put forward an image quality assessment algorithm based on multiscale geometric analysis. The simulation experiments showed that the algorithm improves the performance of image quality evaluation [6].

This paper proposes an image quality evaluation model based on the computation of phase similarity, according to the fact that the human visual system perceives images mainly through the characteristics of low-level image features including edge and phase. This model considers the relationship between the similarity index of phase information and the human visual system. It combines the phase information of images with their structural characteristics, such as brightness, contrast, and edge information, to provide a calculation method of image quality evaluation function.

\section{Phase Characteristic}

Fourier transform provides a frequency-based representation of natural images, which can isolate the phase and amplitude information. When we consider the natural image representation based on frequency, the following questions naturally arise: which is more important, the phase or the amplitude (power)? Or are they equally important? Most researchers believe that the phase information is more important to the perceptual system than the amplitude structure [7]. The quality of the original image is generally poor due to the influence of weather, acquisition distance, and other objective factors. In general, the original image will contain strong noise, poor contrast, and brightness, and the target size will change greatly. Based on image structure or the gradient information processing method, the first step is to remove the image noise of image filtering. However, the average filtering method for multiplicative noise filtering effect is poorer, and the filtering may change the structure of the image or change the location of the object in the image [8]. Quality assessment can be considered to evaluate the visual difference between the test image and the reference image. A quality metric should capture the feature that is closely related to the visual difference. In the Fourier representation of signals, the amplitude and phase information of the spectrum are some of the most important and widely used image representation and analysis tools in spatial frequency conversion. They tend to play different roles, and in some cases many important features of a signal can be retained even if only the phase information is retained [9]. Such a conclusion cannot generally be drawn about spectral amplitude. This discovery of phase has been confirmed in a large number of studies and applications, and it is also true for one-dimensional signals, two-dimensional signals, and three-dimensional signals. For example, sound holograms and optical holograms with only phase and amplitude have been studied. For holograms with only phase (also known as phase diagrams), only the phase of the scattering wave front is recorded, and its amplitude is set as a constant. For holograms with only phase, only the amplitude of the scattering wave front is recorded, and the phase is set as zero [10]. Generally, for the reconstruction of holograms with only amplitude, the reconstructed object is not enough to represent the original object, and for the reconstruction of holograms with only phase, the reconstructed object has many of the same important features as the original object. Closely related to phase- and amplitude-only holograms are phase- and amplitude-only images. Like a phase diagram, a phase-only image has a Fourier transform phase equivalent to the phase of the original image, and the Fourier transform amplitude is a general representation of the spectral amplitude of the image, similar to the average of all unrelated images. It has been proven by some examples that many features of the original image can be clearly identified in the phaseonly image but not in the amplitude-only image. A similar phenomenon occurs in the case of speech signals and crystal Xray images [11]. In particular, for speech, if the phase and unit amplitude of the Fourier transform of a long speech are mixed together, the sentence remains comprehensible. In the case of crystal X-ray images, details of crystal structure can often be inferred from X-ray diffraction data. Only the structure of the correct amplitude of the scattering data and the 
Fourier synthesis of the zero-valued phase cannot generally preserve the structure of the atom; however, only the Fourier synthesis using the correct phase and the unit amplitude can reflect the structure of the atom [12]. These examples strongly suggest the fact that, in many cases, the phase contains more necessary information about the signal. Therefore, the phase information can be the key to identify the image content. Phase information plays an important role in human visual perception systems [13]. Moreover, it has many excellent characteristics and high stability, and it is more in line with human perception characteristics [14]. Thus, phase information is a very important feature for image quality assessment.

\section{The Computation of Phase Similarity Index}

In this section, based on the analysis of phase characteristics, we propose an image quality evaluation model by combining the image brightness information, structural characteristics, and phase information. The proposed quality metric is named the phase similarity index (PSI), the computation of which consists of three components: brightness similarity, contour similarity, and phase similarity.

In practice, this computation is based on image blocks. Firstly, the PSI score of each block is calculated, and then we combine all the PSI scores to evaluate the overall image quality. It is assumed that the reference and the distorted image blocks are represented by $\boldsymbol{x}$ and $\boldsymbol{y}$, respectively. Let $\boldsymbol{b}(\boldsymbol{x}, \boldsymbol{y})$ represent the brightness comparison information of the two images, $\boldsymbol{c}(\boldsymbol{x}, \boldsymbol{y})$ represent the comparison of contour information, and $\boldsymbol{p}(\boldsymbol{x}, \boldsymbol{y})$ denote the comparison results of phase information. Figure 1 is a flowchart of the algorithm proposed in this paper. Details are provided in the following sections.

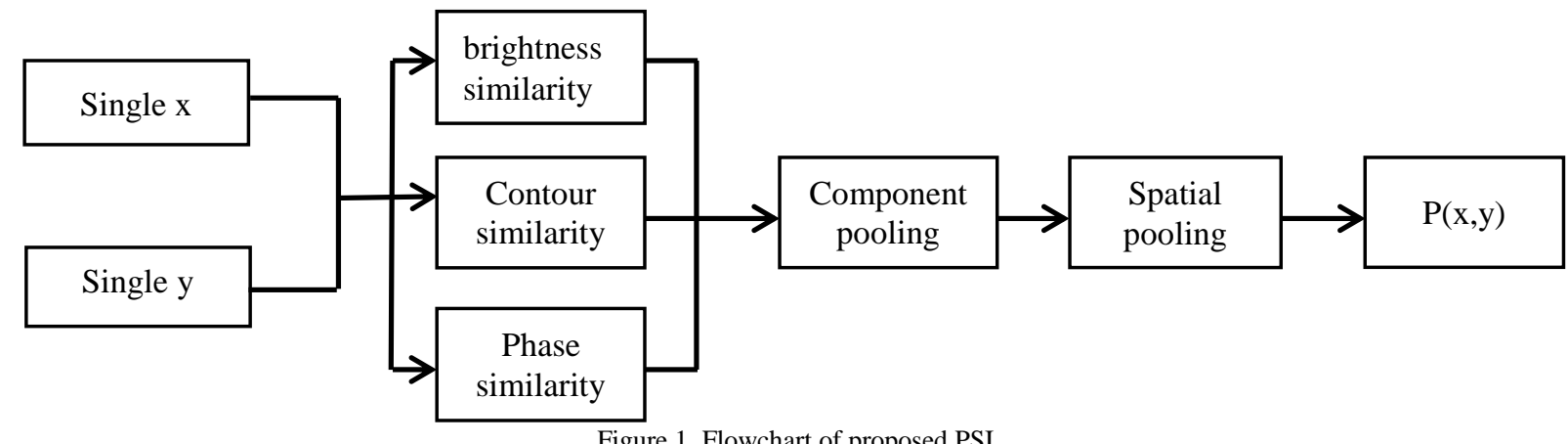

Then, the calculation steps of PSI are as follows. First, compute the following three components: brightness similarity, contour similarity, and phase similarity. Then, use the three components to generate a quality score.

\subsection{Brightness Similarity}

This step aims to calculate the brightness difference of the related two images. Brightness is a fundamental information for images; many image distortions can change the brightness drastically. The influence of ambient brightness on image quality evaluation mainly exists in two aspects: first, the colour phase of the image will shift under the influence of the change of illumination intensity [15], and different colours will shift in different directions and degrees, so the image will change in different degrees and directions due to the influence of illumination intensity. Second, ambient brightness can affect the visual characteristics of human eyes, such as contrast sensitivity, and thus affect the results of image quality evaluation [16]. Therefore, we need to compare the brightness of the image signals during the first step. Assuming that the signal is discrete, we need to calculate an average intensity of a given image. The formula is as follows:

$$
\operatorname{mean}(\boldsymbol{x})=\frac{1}{N} \sum_{i=1}^{N} x_{i}
$$

Where $N$ denotes the total number of pixels in image vector $\boldsymbol{x}$.

Then, the brightness similarity aims to compare mean values of the two image vectors $(\boldsymbol{x}$ and $\boldsymbol{y})$. The calculation formula for the brightness comparison of the two image signals is obtained by the following equation:

$$
b(\boldsymbol{x}, \boldsymbol{y})=\frac{2 \operatorname{mean}(\boldsymbol{x}) \cdot \operatorname{mean}(\boldsymbol{y})+C_{1}}{\operatorname{mean}(\boldsymbol{x})^{2}+\operatorname{mean}(\boldsymbol{y})^{2}+C_{1}}
$$


Where $C_{l}$ is a constant, and its best value is 0.01 .

\subsection{Contour Similarity}

Contour similarity calculates the structure comparison information of the two images. Compared with noise [17], blur, and compression distortion [18], contrast changes cause more image brightness, contrast, and colour distortion [19]. Figure 2 shows the original reference image in Figure 2(a) and two distorted images containing contrast changes, among which the left image in Figure 2(b) is too small to make the colour tone of the picture dim, while the right image in Figure 2(b) is too large to make the picture too bright. As shown in Figure 2(b), the distortion of the image introduced by the contrast change is small, while the intensity, brightness, contrast and colour change are strong enough to make the human eye uncomfortable. Based on this, contrast is considered as a very important feature in the establishment of the image quality evaluation model in this paper.

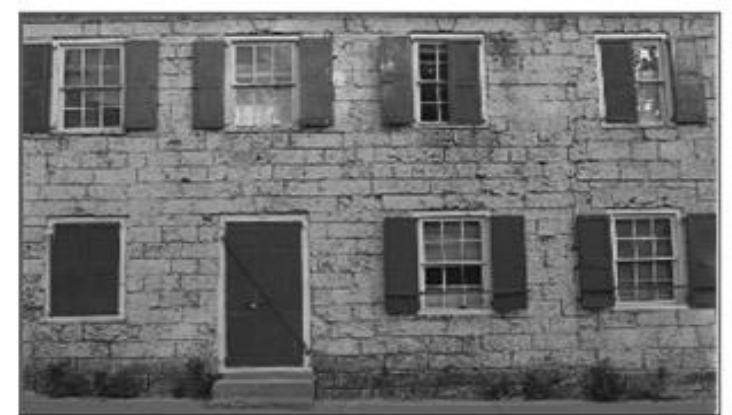

(a) The original image

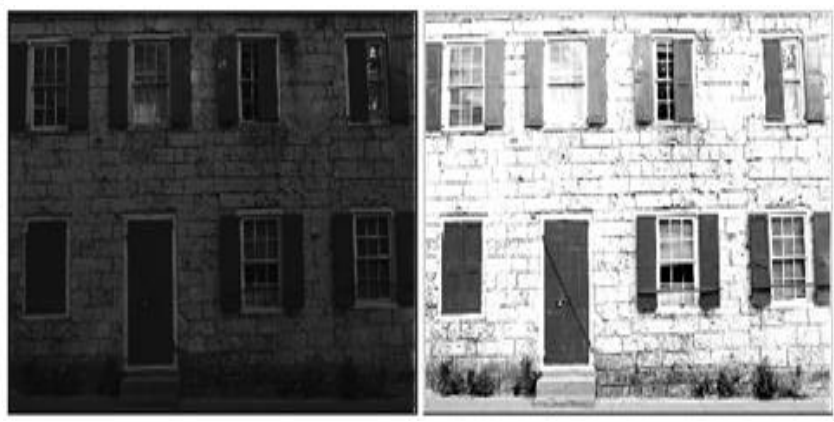

(b) Contrast distorted image

Figure 2. Reference image and contrast-changed distorted image

Then, we remove the average intensity from the relevant image signals. In the discrete form, the obtained signal corresponds to the projection of the vector on the hyperplane, which is defined as follows:

$$
\sum_{i=1}^{N} x_{i}=0
$$

In addition, we need to determine the variance of two related image signals, represented by $v_{x}$ and $v_{y}$. The formula is defined as follows:

$$
v_{x}=\left(\frac{1}{N-1} \sum_{i=1}^{N}\left(x_{i}-\operatorname{mean}(\boldsymbol{x})\right)^{2}\right)^{\frac{1}{2}}
$$

After subtracting the brightness and normalizing the variance, we compare the structure information of the experimental image signals. Specifically, we compare the two unit vectors $(\boldsymbol{x}$ - mean $(\boldsymbol{x}))$ and $(\boldsymbol{y}-\operatorname{mean}(\boldsymbol{y}))$ in the hyperplane defined by Equation (3) with the structure of the two experimental images. Thus, we define the structure comparison function as

$$
c(\boldsymbol{x}, \boldsymbol{y})=\frac{v_{x y}+C_{2}}{v_{x} \cdot v_{y}+C_{2}}
$$

Where $C_{2}$ is a constant, its best value is 11 , and $v_{x y}$ can be obtained by

$$
v_{x y}=\left(\frac{1}{N-1} \sum_{i=1}^{N}\left(x_{i}-\operatorname{mean}(\boldsymbol{x})\right) \cdot\left(y_{i}-\operatorname{mean}(\boldsymbol{y})\right)\right)^{\frac{1}{2}}
$$

\subsection{Phase Similarity}

This component calculates the phase comparison information between two image vectors. Let phase $(\boldsymbol{x})$ denote the phase information of the image vector $\boldsymbol{x}$ after Fourier transform. Then, the phase similarity $(p(\boldsymbol{x}, \boldsymbol{y}))$ can be obtained by the following equation: 


$$
p(\boldsymbol{x}, \boldsymbol{y})=\frac{2 \cdot \operatorname{phase}(\boldsymbol{x}) \cdot \operatorname{phase}(\boldsymbol{y})+C_{3}}{\operatorname{phase}(\boldsymbol{x})^{2}+\operatorname{phase}(\boldsymbol{y})^{2}+C_{3}}
$$

The value of constant $C_{3}$ should be between 0.01 and 0.02 .

\subsection{The Computation of PSI Score}

Finally, the quality score of PSI can be computed by the following equation:

$$
P S I(\boldsymbol{x}, \boldsymbol{y})=b(\boldsymbol{x}, \boldsymbol{y}) \cdot c(\boldsymbol{x}, \boldsymbol{y}) \cdot \beta+p(\boldsymbol{x}, \boldsymbol{y})(1-\beta)
$$

Where $\beta$ is a weighting parameter, and in our experiment its proper value is 0.32 . Then, the quality of an image block can be obtained using the above equation. The quality of the whole image can be obtained by pooling all the blocks together.

$$
\operatorname{PSI}(\mathbf{X}, \mathbf{Y})=\frac{1}{M} \sum_{i=1}^{M} \operatorname{PSI}\left(\boldsymbol{x}_{i}, \boldsymbol{y}_{i}\right)
$$

Where $\mathbf{X}$ and $\mathbf{Y}$ denote the reference and distorted image, respectively, $\boldsymbol{x}_{i}$ and $\boldsymbol{y}_{i}$ denote the block vectors of $\mathbf{X}$ and $\mathbf{Y}$, respectively, and $M$ is the total number of block vectors in the test image.

\section{Experimental Results and Analysis}

In order to test the performance of the PSI method proposed in this paper, we evaluate the image quality results and compare them with the results of other methods. The image data in the image databases CSIQ, TID2008, and TID2013 are used as the research object [20]. The image database TID2008 includes 25 original reference images, and 17 types of distortion processing are used to obtain the distorted images. The intensity of each distortion type included four levels, resulting in a total of 1,700 distorted image data. TID2013 contains 25 reference images and 3,000 distortion images (25 reference images, 24 distortions, and five distortion levels). The image is subjectively evaluated by many observers, and the results of the evaluation are statistically computed to obtain the subjective evaluation score (MOS) [21] of all distorted images, which is used as an objective evaluation scale to measure the image quality [22]. The information for the three databases is listed in Table 1.

Table 1. Characteristics of the three databases for image quality assessment

\begin{tabular}{cccccc}
\hline Database & Distorted images & Reference images & Distortion types & Observers & Image size \\
\hline CSIQ & 866 & 30 & 6 & 35 & $512 \times 512$ \\
TID2008 & 1700 & 25 & 17 & 838 & $512 \times 384$ \\
TID2013 & 3000 & 25 & 24 & 971 & $512 \times 384$ \\
\hline
\end{tabular}

In this paper, the image quality assessment methods for the performance comparison include the PSNR method; SSIM method, which is an image quality evaluation method based on structural similarity; feature structural similarity (FSIM) [23], which is based on the characteristics of the structure similarity [24]; and the method proposed in this paper, which is based on phase information. We compare the four types of methods and analyze the results. These methods are all tested on the CSIQ, TID2008, and TID2013 databases. In order to compare the performance of the algorithm and other algorithms in this paper, VQEG (video quality experts group) [25] is used to obtain the objective evaluation of image measurements and subjective evaluation of images to satisfy certain nonlinear relations. The natural texture and edge information of images are highly unstructured, singularity characteristics; therefore, this paper adopts the following function to define the nonlinear mapping:

$$
f(s)=\alpha_{1}\left(0.5-\frac{1}{1+\exp \left(\alpha_{2}\left(s-\alpha_{3}\right)\right)}\right)+\alpha_{4} s+\alpha_{5}
$$

Figure 3 shows the scatter diagram results of objective evaluation values and the subjective evaluation score MOS of PSNR, SSIM, FSIM, and the method proposed in this paper. In Figure 3, each point represents an image data in the CSIQ database, and the horizontal and vertical coordinate values respectively represent the predicted value of the model and the subjective evaluation score MOS. According to the scatter plot density in Figure 1, the correlation between the predicted value of the image quality model and the subjective evaluation can be intuitively reflected. 
As can be seen from Figure 3, the correlation between the predicted quality value and the subjective evaluation by the PSNR-based evaluation method is poor. The correlation between the predicted quality value and subjective evaluation of the evaluation method based on structural similarity SSIM is significantly improved compared with the PSNR method. However, when the SSIM method has severe image distortion, that is, when the SSIM value is small, the correlation between the value predicted by the objective evaluation method and the subjective evaluation is poor. According to the scatter plot in Figure 3(b), the distribution of points on the scatter plot is scattered. The FSIM method based on the similarity of feature structure has a good prediction effect, and the points in the scatter plot are relatively dense. The prediction quality of the method in this paper has a good correlation with the subjective evaluation. Its performance is better than that of the PSNR and SSIM methods and equivalent to that of the FSIM method.

In order to demonstrate the relationship between the quality and distortion types, Figure 4 shows the fitting curves of different distortion types for the three best image quality metrics (i.e., SSIM, FSIM, and PSI) in our experiment.

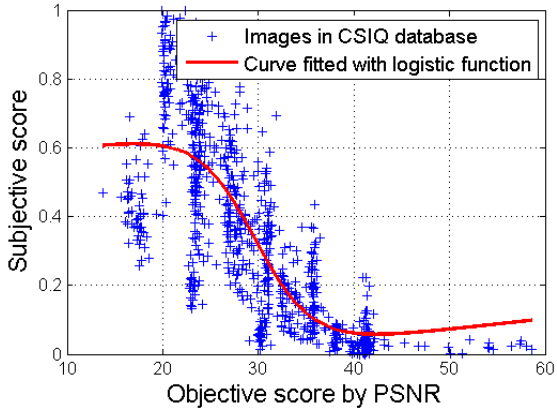

(a)

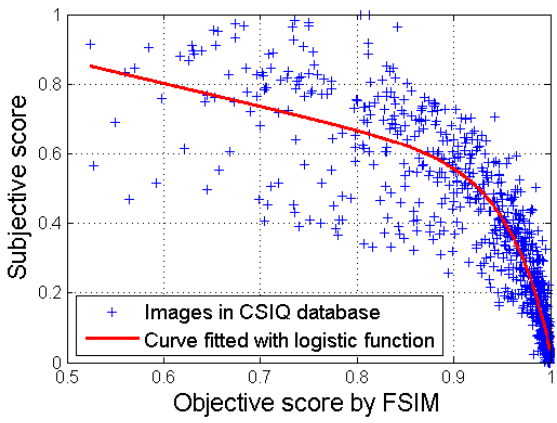

(c)

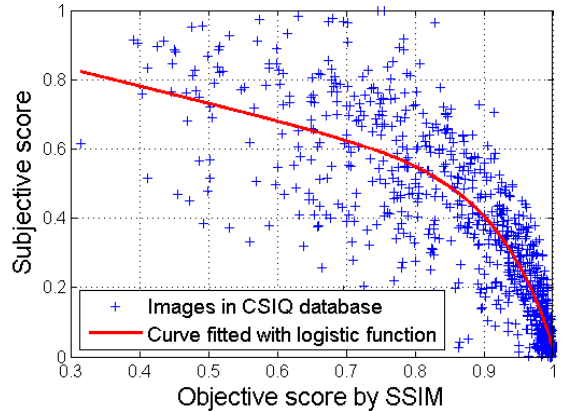

(b)

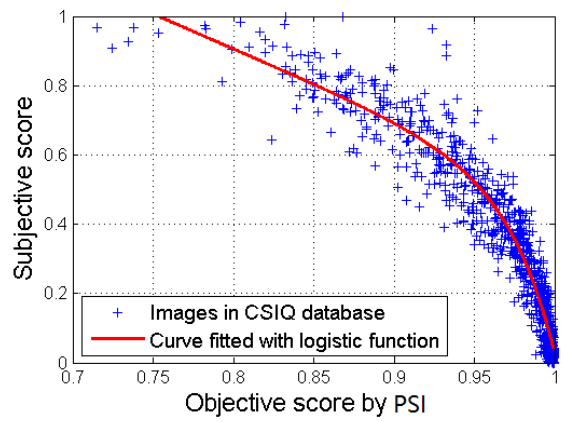

(d)

Figure 3. Scatter plot of the predicted value of the distorted image data model compared with the subjective evaluation scores on CSIQ database

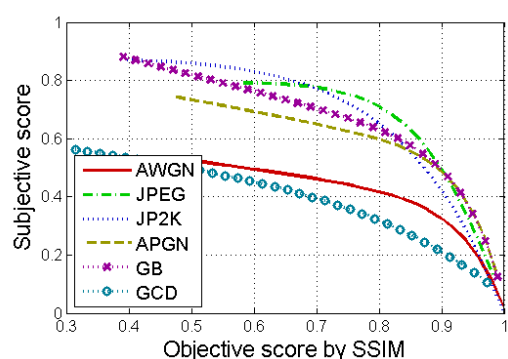

(a)

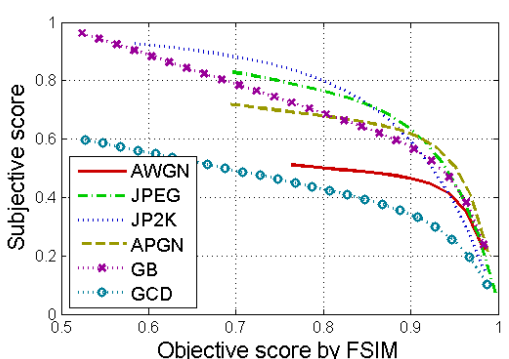

(b)

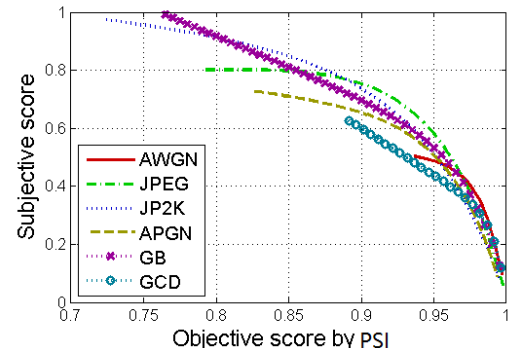

(c)

Figure 4. Fitting curves of different distortions for the three best IQMs on CSIQ database

In order to further verify the image quality evaluation method proposed in this paper and compare its performance with the existing methods, this paper selects the reference standard of the image quality evaluation model provided by VQEG. The Pearson linear correlation coefficient (PLCC), root mean square error (RMSE), Kendall rank order correlation coefficient (KROCC), Spearman rank correlation coefficient (SROCC), and outlier ratio (OR) are selected to test the performance of various evaluation methods [26].

The Pearson linear correlation coefficient is mainly used to test the correlation between subjective evaluation and objective evaluation to predict the accuracy of image quality evaluation performance [27]. The larger the Pearson linear 
correlation coefficient, the better the prediction of the image quality evaluation model. KROCC is similar to SROCC and can be used to measure the nonlinear and linear correlation of the model [28]. Root mean square error represents the mean square error between the objective evaluation value and the subjective quality score. The smaller the value, the better the prediction model of image quality. The Spearman rank correlation coefficient measures the degree of agreement between the objective evaluation (predicted value) and the subjective evaluation value in terms of amplitude. OR reflects whether the image prediction model can accurately predict the quality of all types of image quality. It is defined as the ratio of the number of points (also known as departure points) whose prediction error exceeds a certain threshold to the number of all points. The smaller the departure rate, the better the prediction of the proposed evaluation model.

Tables 2 and 3 show the performance and quality comparison results of several image quality evaluation methods. As can be seen from Table 2, the method in this paper has a good evaluation performance. Table 2 lists the performance comparison results of several image quality evaluation algorithms on the TID2008 image database to test all the distorted image data. Table 3 lists the performance comparison results of several image quality evaluation algorithms on the TID2013 image database to test all the distorted image data. According to the results of simulation experiments, the four indexes of the algorithm in this paper are generally better than those of the PSNR and SSIM methods, and the performance of the FSIM method proposed in literature is comparable. As can be seen from the experimental results in Tables 2 and 3 , the phase characteristics and structural characteristics of the image itself are comprehensively considered in this paper. The PSI image quality evaluation algorithm proposed in this paper has a clear physical significance and is more in line with the subjective perception characteristics of the image quality of the human visual system.

Table 2. TID2008 image database several objective evaluation algorithm performance comparison

\begin{tabular}{|c|c|c|c|c|c|}
\hline IQA model & SROCC & KROCC & PLCC & RMSE & OR \\
\hline PSNR & 0.5531 & 0.4027 & 0.5734 & 1.0994 & 0.1320 \\
\hline SSIM & 0.7749 & 0.5768 & 0.7732 & 0.8511 & 0.0334 \\
\hline FSIM & 0.8805 & 0.6946 & 0.8738 & 0.6525 & 0.0217 \\
\hline PSI & 0.8965 & 0.7183 & 0.9011 & 0.6496 & 0.0214 \\
\hline IQA model & SROCC & KROCC & PLCC & RMSE & OR \\
\hline PSNR & 0.5729 & 0.4122 & 0.5334 & 1.0772 & 0.1427 \\
\hline SSIM & 0.7417 & 0.5588 & 0.7895 & 0.7068 & 0.0384 \\
\hline FSIM & 0.8015 & 0.6289 & 0.8589 & 0.6349 & 0.0232 \\
\hline PSI & 0.8979 & 0.7127 & 0.8762 & 0.6466 & 0.0211 \\
\hline
\end{tabular}

\section{Conclusions}

Phase information is a very important feature that can distinguish between distorted images and reference images. Many quality assessment models do not take into account the phase characteristics of the natural image itself, and the correlation between the quality predicted by the model and the subjective evaluation value is not high. Therefore, in order to solve this problem, on the basis of brightness and contour information, this paper further considered the characteristics of image phase similarity and proposed an image quality evaluation model called the phase similarity index. This model is simple to implement and can effectively predict the subjective perception of image quality. The simulation results on the CSIQ, TID2008, and TID2013 image databases showed that, compared with PSNR and SSIM, the image quality algorithm proposed in this paper can better predict the performance and images more consistently with the subjective perception of human eyes, and the correlation between the objective forecast value and the subjective evaluation is better. At the same time, the performance of the prediction model is similar to that of the prediction method based on feature structure similarity. In terms of application, the proposed image quality evaluation model can check the image quality status and video data flow resources in the digital video service network and optimize the configuration of the video image bit allocation algorithm according to the results of the evaluation model.

\section{Acknowledgements}

This research is supported by the National Natural Science Foundation of China (No. 61401404), the Key Research Project of Colleges in Henan Province (No. 15A520107), and the Key Scientific and Technological Program in Henan Province (No. 192102210136).

\section{References}

1. Z. Wang and Q. Li, "Information Content Weighting for Perceptual Image Quality Assessment," IEEE Transactions on Image Processing, Vol. 20, No. 5, pp. 1185-1198, May 2011 
2. Z. Wang, A. C. Bovik, H. R. Sheikh, and E. P. Simoncelli, "Image Quality Assessment: From Error Visibility to Structural Similarity," IEEE Transactions on Image Processing, Vol. 13, No. 4, pp. 600-612, April 2004

3. Z. Wang and A. C. Bovik, "Mean Squared Error: Love It or Leave It? A New Look at Signal Fidelity Measures," IEEE Signal Processing Magazine, Vol. 26, No. 1, pp. 98-117, January 2009

4. M. P. Sampat, Z. Wang, S. Gupta, A. C. Bovik, and M. K. Markey, "Complex Wavelet Structural Similarity: A New Image Similarity Index," IEEE Transactions on Image Processing, Vol. 18, No. 11, pp. 2385-2401, 2009

5. D. M. Chandler and S. S. Hemami, "VSNR: A Wavelet-based Visual Signal-to-Noise Ratio for Natural Images," IEEE Transactions on Image Processing, Vol. 16, No. 9, pp. 2284-2298, September 2007

6. X. Gao, W. Lu, D. Tao, and X. Li, "Image Quality Assessment based on Multiscale Geometric Analysis," IEEE Transactions on Image Processing, Vol. 18, No. 7, pp. 1409-1423, July 2009

7. A. Liu, W. Lin, and M. Narwaria, "Image Quality Assessment based on Gradient Similarity," IEEE Transactions on Image Processing, Vol. 21, No. 8, pp. 1500-1512, April 2012

8. W. F. Xue, L. Zhang, X. Q. Mou, and A. Bovik, "Gradient Magnitude Similarity Deviation: A Highly Efficient Perceptual Image Quality Index," IEEE Transactions on Image Processing, Vol. 23, No. 3, pp. 684-695, February 2014

9. M. C. Morrone, J. Ross, D. C. Burr, and R. Owens, "Mach Bands are Phase Dependent," Nature, Vol. 324, No. 6049, pp. 250253, November 1986

10. M. C. Morrone and D. C. Burr, "Feature Detection in Human Vision: A Phase-Dependent Energy Model," Proc. R. Soc. Lond.B, Vol. 235, No. 1280, pp. 221-245, December 1988

11. M. C. Morrone and R. A. Owens, "Feature Detection from Local Energy," Pattern Recognit Letters, Vol. 6, No. 5, pp. 303-313, December1987

12. P. Kovesi, "Image Features from Phase Congruency," Videre: J. Comp. Vis. Res, Vol. 1, No. 3, pp. 1-26, November 1999

13. C. C. Yang and S. H. Kwok, "Efficient Gamut Clipping for Color Image Processing using LHS and YIQ," Opt. Eng, Vol. 3, No. 42, pp. 701-711, 2003

14. N. N. Ponomarenko, L. Jin, O. Ieremeiev, V. V. Lukin, K. Egiazarian, J. T. Astola, et al., "Image Database TID2013: Peculiarities, Results and Perspectives," Sig. Process: Image Commun, Vol. 7, No. 30, pp. 57-77, 2015

15. D. Kelly, "Motion and Vision I: Stabilized Images of Stationary Gratings," J. Opt. Soc. Amer., Vol. 69, No. 9, pp. 1266-1274, 1979

16. E. C. Larson and D. M. Chandler, "Most Apparent Distortion: Full-Reference Image Quality Assessment and the Role of Strategy," J. Electron. Imaging, Vol. 19, No. 1, pp. 011006:1-011006:21, 2010

17. X. Ran and N. Farvardin, "A Perceptually Motivated Three-Component Image Model-Part I: Description of the Model," IEEE Trans. Image Process, Vol. 4, No. 4, pp. 401-415, April 1995

18. E. Ong, W. Lin, Z. Lu, S. Yao, and M. Etoh, "Visual Distortion Assessment with Emphasis on Spatially Transitional Regions," IEEE Trans. Circuits Syst. Video Technol, Vol. 14, No. 4, pp. 559-566, April 2004

19. Z. Wang and E. P. Simonceclli, "Translation Insensitive Image Similarity in Complex Wavelet Domain," in Proceedings of Int. Conference Acoust., Speech, Signal Process, Vol. 12, No. 21, pp. 573-576, 2005

20. C. Yang, W. Gao, and L. Po, "Discrete Wavelet Transform-based Structural Similarity for Image Quality Assessment," in Proceedings of Int. Conference Image Process, pp. 377-380, 2008

21. G. Chen, C. Yang, and S. Xie, "Edge-based Structural Similarity for Image Quality Assessment," in Proceedings of Int. Conference Acoust., Speech, Signal Process, pp. 14-19, 2006

22. A. Ahar, A. Barri, and P. Schelkens, "From Sparse Coding Significance to Perceptual Quality: A New Approach for Image Quality Assessment," IEEE Transactions on Image Processing, Vol. 27, No. 2, pp. 879-893, February 2018

23. L. Zhang, L. Zhang, X. Mou, and D. Zhang, "FSIM: A Feature Similarity Index for Image Quality Assessment," IEEE Transactions on Image Processing, Vol. 8, No. 20, pp. 2378-2368, 2011

24. L. Zhang, L. Zhang, X. Mou, and D. Zhang, "FSIM: A Feature Similarity Index for Image Quality Assessment," IEEE Transactions on Image Processing, Vol. 20, No. 8, pp. 2378-2386, August 2011.

25. "VQEG. Final Report from the Video Quality Experts Group on the Validation of Objective Models of Video Quality Assessment," (http:// www.vqeg.org/)

26. H. Sheikh and A. C. Bovik, "Image Information and Visual Quality," IEEE Trans. Image. Process, Vol. 4, No. 13, pp. 600-612, 2006

27. L. Zhang, Y. Shen, and H. Y. Li, "VSI: A Visual Saliency Induced Index for Perceptual Image Quality Assessment," IEEE Trans. Image. Process, Vol. 10, No. 23, pp. 4270-4281, 2014

28. K. Gu, G. T. Zhai, X. K. Yang, W. J. Zhang, and M. Liu, "Subjective and Objective Quality Assessment for Images with Contrast Change," IEEE Int. Conference Image Processing, pp. 383-387, 2008 